How does socio-economic position (SEP) get biologically embedded? A comparison of allostatic load and the epigenetic clock(s)

\title{
McCrory, Cathal
}

2019-06

McCrory , C , Fiorito , G , Cheallaigh , C N , Polidoro, S, Karisola , P , Alenius , H , Layte , R , Seeman , T , Vineis , P \& Kenny , R A 2019, ' How does socio-economic position (SEP) get biologically embedded? A comparison of allostatic load and the epigenetic clock(s) ' , Psychoneuroendocrinology, vol. 104 , pp. 64-73 . https://doi.org/10.1016/j.psyneuen.2019.02.018

http://hdl.handle.net/10138/317092

https://doi.org/10.1016/j.psyneuen.2019.02.018

publishedVersion

Downloaded from Helda, University of Helsinki institutional repository.

This is an electronic reprint of the original article.

This reprint may differ from the original in pagination and typographic detail.

Please cite the original version. 


\title{
How does socio-economic position (SEP) get biologically embedded? A comparison of allostatic load and the epigenetic clock(s)
}

\author{
Cathal McCrory $^{\mathrm{a}, 1, *}$, Giovanni Fiorito ${ }^{\mathrm{b}, 1}$, Cliona Ni Cheallaigh ${ }^{\mathrm{a}}$, Silvia Polidoro ${ }^{\mathrm{b}}$, Piia Karisola ${ }^{\mathrm{c}}$, \\ Harri Alenius ${ }^{\mathrm{c}, \mathrm{d}}$, Richard Layte ${ }^{\mathrm{e}}$, Teresa Seeman ${ }^{\mathrm{f}}$, Paolo Vineis ${ }^{\mathrm{g}}$, Rose Anne Kenny ${ }^{\mathrm{a}}$ \\ ${ }^{a}$ The Irish Longitudinal Study on Ageing, Trinity College Dublin, Ireland \\ ${ }^{\mathrm{b}}$ Italian Institute for Genomic Medicine (IIGM, former HuGeF), Italy \\ ${ }^{\mathrm{c}}$ Faculty of Medicine, University of Helsinki, 00014, Helsinki, Finland \\ ${ }^{\mathrm{d}}$ Institute of Environmental Medicine (IMM), Karolinska Institutet, Stockholm, Sweden \\ e Department of Sociology, Trinity College Dublin, Ireland \\ ${ }^{\mathrm{f}}$ David Geffen School of Medicine, UCLA, USA \\ ${ }^{\mathrm{g}} \mathrm{MRCPHE}$ Centre for Environment and Health, Imperial College London, United Kingdom
}

\section{A R T I C L E I N F O}

\section{Keywords:}

Socioeconomic position

Epigenetic clock

Allostatic load

Biological embedding

Life Course

\begin{abstract}
A B S T R A C T
Individuals of lower socio-economic position (SEP) carry a heavier burden of disease and morbidity and live shorter lives on average compared with their more advantaged counterparts. This has sparked research interest in the processes and mechanisms via which social adversity gets biologically embedded. The present study directly compares the empirical worth of two candidate mechanisms: Allostatic Load (AL) and the Epigenetic Clock(s) for advancing our understanding of embodiment using a sub-sample of 490 individuals from the Irish Longitudinal Study (TILDA) who were explicitly selected for this purpose based on their inter-generational life course social class trajectory. A battery of 14 biomarkers representing the activity of 4 different physiological systems: Immunological, Cardiovascular, Metabolic, and Renal was used to construct the AL score. Biomarkers were dichotomised into high and low risk groups according to sex-specific quartiles of risk and summed to create a count ranging from 0-14. Three measures of epigenetic age acceleration were computed according to three sets of age-associated Cytosine-phosphate-Guanine $(\mathrm{CpG})$ sites described by Horvath, Hannum and Levine. AL was strongly socially patterned across a number of measures of SEP, while the epigenetic clocks were not. AL partially mediated the association between measures of SEP and an objective measure of physiological functioning: performance on the Timed Up and Go (TUG test). We conclude that AL may represent the more promising candidate for understanding the pervasive link between SEP and health.
\end{abstract}

\section{Introduction}

One of the most consistent findings in epidemiological research is that health is socially patterned and that individuals in lower socioeconomic strata will experience a heavier burden of disease across the life course (Marmot et al., 2012). On average, they will develop diseases earlier and die younger compared with their more advantaged peers (Stringhini et al., 2017). Because the risk of poor health tends to decline with step increases in socio-economic position (SEP), the relationship has come to be known as the social gradient in health. The robustness of these associations and the fact that SEP seems to be important for just about every disease outcome studied suggests that there may be a common cause/mechanism underlying the pathogenesis of different disease types. For example, chronic stress can damage biological systems directly (e.g. circulating levels of glucocorticoids) as well as indirectly via its effects on other biobehavioural risk factors (e.g. smoking, alcohol consumption, sleep quality) that are known to impinge upon health (McEwen, 2008).

While much of the early work in this field was characterized by heated debate concerning whether material or psychosocial explanations had greater explanatory power in accounting for the social gradient in health (Lynch et al., 2000; Marmot and Wilkinson, 2001), a less entrenched position is simply to acknowledge that both material and psychosocial privation can wreak havoc at the biological level. Hence the conceptual model that informs our approach views SEP as a measure that includes one or more of a range of risk exposures: material,

\footnotetext{
* Corresponding author.

E-mail address: mccrorc@tcd.ie (C. McCrory).

${ }^{1}$ Joint first authors.
} 
psychosocial, and environmental that predispose to disease through a number of potential pathways (Adler and Stewart, 2010). Accepting this, the challenge then is to identify the mechanisms through which life course social influences get transduced at the biological level to erode health. Krieger (2005) refers to this process as "embodiment", Hertzman (2012) as bioarcheology, while Taylor et al. (1997) ponder how social adversity "gets under the skin". A number of candidate processes and mechanisms have been proposed that provide intermediate frameworks linking SEP and health including, telomere shortening (Robertson et al., 2012), inflammation (Aiello and Kaplan, 2009), epigenetic modifications (Cunliffe, 2016), and allostatic load (Seeman et al., 2004). A number of criteria have to be satisfied for any candidate process to make a claim to mediate the association of SEP with health. The mediator must be structured according to SEP, the mediator must be correlated with health, and the addition of the mediator should lead to a statistically significant reduction in the magnitude of the SEP-health relationship. In this paper we consider two of these candidate processes: allostatic load (AL) and epigenetic age acceleration (EAA).

\subsection{Allostatic load}

It has been proposed that cumulative lifetime exposure to material, social, psychological and/or environmental stressors increases the risk of diseases in later life by disrupting the physiological regulatory systems that are involved with initiating, maintaining, and inhibiting the stress response leading to greater 'wear and tear' on the body (Seeman, Epel, Gruenewald et al., 2010). Indeed, a large body of evidence has accrued showing that social gradients are evident across a large number of biological parameters that are implicated in the stress response including cortisol secretion, norepinephrine, epinephrine, heart rate variability, and inflammatory markers (Seeman et al., 2010). Researchers have attempted to capture variation in these life-course stresses using an AL score, which is a multi-system, multi-dimensional composite index, usually involving neuroendocrine, immunological, cardiovascular, and metabolic components - that has been used to quantify stress-induced biological risk (Seeman et al., 2010). AL is socially patterned (Gruenewald et al., 2012; Seeman et al., 2014; Delpierre et al., 2016) and predicts age-related diseases, frailty, and mortality independently of age and other risk factors (Karlamangla et al., 2002; Castagné et al., 2018).

A handful of studies have examined the association of SEP with AL over the life course (Gustafsson et al., 2011; Gruenewald et al., 2012; Robertson et al., 2014; Solis et al., 2016; Prag and Richards, 2018). Comparison of results across studies is complicated by the fact that $\mathrm{AL}$ is not always measured using the same number or composition of biomarkers, but most of them have concluded that the socio-economic conditions of childhood and adulthood have separable and additive effects on AL. Gruenewald et al. (2012) reported significant effects of childhood, and adulthood SEP on AL using the MIDUS cohort, but the magnitude of the effect size was about twice as large for adulthood compared with childhood SEP. They also observed a dose-response relation between SEP disadvantage score and AL, and a gradient with life course mobility such that the persistent low SEP had the highest AL score followed by the downwardly mobile, then the upwardly mobile, while the persistent high SEP had the lowest score. Robertson et al. (2014) examined the association of SEP with AL across 3 life stages (childhood, transition to adulthood, and adulthood) in 3 age cohorts and compared the goodness of fit of 3 life course models (critical periods, accumulation, social mobility) that have been advanced to explain the social patterning of health using data from the West of Scotland Twenty-07 study. Accumulated SEP was the strongest predictor of allostatic load in two of the 3 cohorts (1970's and 1950's, but not the 1930 's cohort). In the 1970's cohort, childhood SEP was also found to be more strongly associated with AL than adulthood SEP. Gustafsson et al., 2011 reported bivariate associations of SEP with AL across 4 stages of the life course $(16,21,30$ and 43 years of age) in a Swedish cohort, but only the most recent measure of SEP was significant when all stages were entered simultaneously in the same regression model, suggesting that early life SEP is important because it helps determine (and may place constraints) on adulthood SEP. A very recent study using Understanding Society concluded that the effects of childhood and adulthood SEP on AL were comparable (Prag and Richards, 2018).

\subsection{Epigenetics}

Variation in gene expression as opposed to gene sequencing is the key concept within the study of epigenetics. It involves chemical modifications to DNA that change the way in which genes are expressed without changing the underlying genetic structure. Interest in the epigenome has grown rapidly in recent years because it is exquisitely plastic - particularly in early life - can be programmed or reprogrammed by environmental experience (Francis, 2009); and represents a potential mechanism via which social exposures occurring in early life are embodied at the molecular level, affecting phenotypic expression. Although many types of epigenetic processes have been identified, the one most readily researched in the context of SEP has been DNA methylation (DNAm) (Majnik and Lane, 2014). One of the most novel and exciting applications of DNAm data is in the development of the so called "Epigenetic Clocks".

\subsection{Epigenetic clocks}

Horvath (2013) developed a multi-tissue predictor that allows the age of most tissues and cell types to be estimated based on DNAm at 353 age-associated CpG sites, while Hannum et al. (2013) developed a blood-specific DNAm age predictor based on levels at $71 \mathrm{CpG}$ sites. These clocks track chronological age very closely $(r=0.90-0.95$ in a sample with a full age range) and allow estimation of whether an individual is experiencing accelerated or decelerated ageing by defining EAA as the difference between DNAm age and chronological age. A positive residual describes an individual experiencing accelerated ageing while a negative residual indicates an individual who is ageing more slowly than their chronological age. Both Horvath's and Hannum's epigenetic clocks were developed to predict the chronological age with the maximum possible accuracy. In both cases, the set of CpGs were selected by elastic net regression using chronological age as the outcome. The only difference between the two is that Horvath's clock works well for all tissues whereas Hannum's clock works well on blood samples only. Levine's epigenetic clock (Levine et al., 2018), which is the first of the 'second generation' clocks, was developed using a slightly different approach. Specifically, a two-step algorithm was implemented: i) a set of ten biomarkers (albumin, creatinine, glucose, creactive protein, lymphocyte percent, mean cell volume, red cell distribution width, alkaline phosphatase, white blood cell count and chronological age) strongly predictive of all-cause mortality were selected and used to estimate the individuals' phenotypic age; ii) elastic net regression was then used to select $513 \mathrm{CpGs}$ using the previously described phenotypic age as the outcome.

Although, both Horvath and Hannum measures exhibit statistically significant associations with many age-related diseases and conditions, the effect sizes are usually small. This is because the use of chronological age as the reference favours the selection of CpGs having timedependent changes, excluding those signals determining the difference between chronological and biological age. Levine's epigenetic clock was built to address this issue, and this is the reason why it outperforms 'first generation clocks' concerning the prediction of many chronic conditions. These epigenetic clocks have been shown to be associated with a range of age-related diseases, functional impairments, and mortality (Horvath and Raj, 2018). Although the clocks are a relatively recent development, early studies provide at least some tentative support for the idea that epigenetic processes may be implicated in the 
earlier ageing of socially disadvantaged groups.

A study from the LIFEPATH group involving data for more than 5000 individuals in Italy, Australia and Ireland found that lower socioeconomic rank was associated with EAA (Fiorito et al., 2017). In the meta-analysis of these cohorts, the epigenetic age of low SEP individuals was estimated to be 1-2 years higher compared with the highest SEP group. The LIFEPATH study also examined the impact of different life course trajectories on EAA by cross-classifying childhood and adulthood social class, finding that the persistent low SEP group were epigenetically older than the persistent high SEP group, with the upwardly and downwardly mobile groups ranking somewhere in between, although this did not amount to a statistically significant difference.

Two more recent studies that explicitly examined the impact of life course SEP on epigenetic ageing found that childhood SEP, but not adulthood SEP was associated with EAA. Austin et al. (2018) examined the association of life course SEP with epigenetic ageing of monocytes among a sample of 335 Canadian participants aged 15-55 years who were selected on the basis of their life course socio-economic trajectory into four groups: stable low, upwardly mobile, stable high, downwardly mobile. They found that participants who experienced low early life SEP (i.e. parental social class before the age of 5) were significantly epigenetically older (0.97-3 years) than those who experienced high SEP in early life, but there was no main effect of adulthood SEP, and the childhood*adulthood interaction was also non-significant. Hughes et al. (2018) examined the association between a variety of contemporaneous and historic measures of SEP and DNAm age, indexed using the Horvath and Hannum clocks, among a sample of 1099 participants aged 18-99 years in the United Kingdom. They observed no significant associations of adulthood SEP (i.e. current income, employment status, cumulative income and employment over a 12-year period) with the epigenetic clocks, but they did report significant associations between a measure of childhood SEP (i.e. father's social class before the age of 16) and EAA adjusting for age sex, white blood cell count, smoking and body mass index.

The present study examines the association of a number of measures of childhood and adulthood SEP with AL and three Epigenetic Clocks (Horvath's, Hannum's, Levine's) among a sub-sample of 490 TILDA participants who were chosen specifically on the basis of their life course socio-economic trajectory. The study contributes to the existing literature in a number of important ways. We directly compare the utility of AL and the Epigenetic Clocks for advancing our understanding of the biological embedding of socio-economic adversity in the same cohort of participants. Secondly, we examine the association of life course SEP with a recently developed second-generation epigenetic clock (Levine's DNAm Phenoage) (Levine et al., 2018), which has been shown to outperform the first-generation clocks in the prediction of many age-related diseases. To the best of our knowledge, this is the first study to examine the association of SEP with Levine's clock.

\section{Method}

\subsection{Sample}

The Irish Longitudinal Study on Ageing (TILDA) is a large prospective cohort study examining the social, economic, and health circumstances of 8175 community-dwelling older-adults aged 50 years of age and older resident in the Republic of Ireland. A detailed description of study design is available elsewhere (Whelan and Savva, 2013). Briefly, respondents completed a computer-assisted personal interview ( $n=8175)$ at home. All participants were subsequently invited to undergo a comprehensive clinical health assessment at one of two national centers using trained nursing staff and standard operating protocols. This analysis uses a sub-sample $(n=490)$ of the TILDA cohort who were selected based on their life-course socio-economic trajectory (described below) and for whom DNA methylation age was available.

\subsection{Measurement of allostatic load}

A battery of 14 biomarkers representing the activity of 4 different physiological systems: Immunological (C Reactive Protein (CRP), Interleukin 1 receptor-antagonist (IL1ra), Interleukin 6 (IL6), Interleukin 8 (IL8)), Cardiovascular (Systolic Blood Pressure (SBP), Diastolic Blood Pressure (DBP), Resting Heart Rate (RHR)), Metabolic (High Density Lipoprotein (HDL), Total cholesterol (TC), Waist-hip ratio (WHR), Body Mass Index (BMI), Glycated haemoglobin (HbA1c)), and Renal (Creatinine, Cystatin C) was used to construct the AL score. An overall AL score was calculated by summing the number of parameters for which a respondent fell within the highest risk quartile using sex-specific cut-offs (range $=0-14$ ). Following Seeman et al. (2014), we incorporated medication data into the calculation of our AL score. Medication use was recorded during the course of the household interview and confirmed by cross-checking the labels on the medicinal packaging. The international non-proprietary name (INN) for any regularly taken medications was assigned and coded using Anatomic Therapeutic Classification Codes (ATC). Participants were classified as high risk in SBP if they were taking anti-hypertensive medication (C02, C03, C09), high risk in RHR if they were taking beta-blockers (C07) or calcium channel blockers (C08), high risk in glycated haemoglobin if taking any diabetes medications, including insulin (A10), and high risk in cholesterol if taking statins (C10AA, C10BA, C10BX). Participants taking these medications were included in addition to the highest risk sex-specific quartile cut-off. As a sensitivity check to ensure that any observed relationship of AL with SEP was not simply being driven by the inclusion of those on medications, we also calculated a second measure of AL that ignores the medication data. We present both sets of results for comparative purposes. The overall level of missingness with respect to the AL biomarkers was very small. Only five people were missing on any biomarker and the maximum number of biomarkers for which any one person was missing was three or less. Following Castagné et al. (2018), a conservative approach (maximum bias imputation) was taken to those missing on any biomarker; by systematically classifying them as 'not at risk' if missing on the biomarker.

C-Reactive Protein (CRP) was measured using ELISA Kit (Cat No CYT298 Millipore), with sensitivity of $0.20 \mathrm{ng} / \mathrm{ml}$. A control serum from one donor and commercially available LiCheck control (Bio-Rad, Ref. 591-596) were used as controls on all the plates. The inflammatory markers, Interleukin 1 receptor-antagonist, Interleukin 6 and Interleukin 8 were measured by Luminex (Bio-Plex 200, Bio-Rad), and spiked serum as well as two concentrations of known samples were used as controls on each plate. Two measurements of seated SBP, DBP and RHR were obtained separated by a 1-min interval using an automatic digital BP monitor (OMRONTM, M10-IT). The means of the two readings were averaged to derive SBP, DBP and RHR. Respondents provided a nonfasting blood sample during the course of the health assessment and these were sent for immediate analysis (within $24 \mathrm{~h}$ ) to derive a detailed lipid profile which included HDL, and TC. BMI was calculated from measured height and weight. Height was measured using a SECA 240 wall mounted measuring rod and weight was measured using a SECA electronic floor scales. WHR is a measure of distribution of body fat (both subcutaneous and intra-abdominal). The waist was defined as the point midway between the iliac crest and the costal margin (lower rib). The hip circumference was defined as being the widest circumference over the buttocks and below the iliac crest. HbA1c was analysed by reversed-phase cation exchange chromatography using an ADAMS HA-8180V analyser which is traceable to the internationally agreed standard developed by the International Federation of Clinical Chemistry. Cystatin C and Creatinine were measured simultaneously from frozen plasma. Cystatin $\mathrm{C}$ was measured using a second generation particle enhanced immunoturbidimetric assay (Roche Tina-quant ${ }^{\mathrm{TM}}$ ) on a Roche Cobas 701 analyzer. This assay has a measuring range of $0.40-6.80 \mathrm{mg} / \mathrm{L}$ and is traceable to the European reference standard material (ERM-DA471/IFCC) for Cystatin C. Creatinine was measured 
using an enzymatic method traceable to isotope-dilution mass spectrometry (Roche Creatinine plus ver.2, Roche Diagnostics, Basel Switzerland).

\subsection{Measurement of epigenetic age acceleration}

For the microarray, DNA samples were extracted from buffy coats using the QIAGEN GENTRA AUTOPURE LS (Qiagen, Crawley, UK). Bisulphite conversion of $500 \mathrm{ng}$ of each sample was performed using the EZ DNA Methylation-Lightning ${ }^{\mathrm{TM}}$ Kit according to the manufacturer's protocol (Zymo Research, Orange, CA). Then, bisulfite-converted DNA was used for hybridization on the Infinium HumanMethylation 850k BeadChip, following the Illumina Infinium HD Methylation protocol. Briefly, a whole genome amplification step was followed by enzymatic end-point fragmentation and hybridization to HumanMethylation EPIC Chip at $48^{\circ} \mathrm{C}$ for $17 \mathrm{~h}$, followed by single nucleotide extension. The incorporated nucleotides were labeled with biotin (ddCTP and ddGTP) and 2,4-dinitrophenol (DNP) (ddATP and ddTTP). After the extension step and staining, the BeadChip was washed and scanned using the Illumina HiScan SQ scanner. The intensities of the images were extracted using the GenomeStudio (v.2011.1) Methylation module (1.9.0) software, which normalizes within-sample data using different internal controls that are present on the HumanMethylation 850k BeadChip and internal background probes. The methylation score for each CpG was represented as a $\beta$-value according to the fluorescent intensity ratio representing any value between 0 (unmethylated) and 1 (completely methylated).

DNA methylation age was computed according to the algorithm described by Horvath (Horvath, 2013), based on a set of 353 age-associated CpG sites, the one based on 71 blood-specific age-associated CpG sites described by Hannum et al.(2013), and the one based on the 513 CpG sites described by Levine et al. (2018). Out of the 889 agerelated CpGs, (6 are in common between Horvath and Hannum, 41 between Horvath and Levine, and 6 between Levine and Hannum) we detected 867 (more than 96\%). The CpGs missing are those that are not present in the new Illumina 850k methylation BeadChip. Briefly, the DNA methylation age is computed as a weighted average of the age-related CpGs, with weights defined using a penalized regression model (Elasticnet regularization) (Horvath, 2013). The few missing values were imputed using the k-nearest neighboring (KNN) imputation algorithm implemented in the R Bioconductor package impute (Troyanskaya et al., 2001). Epigenetic Age Acceleration (EAA) was defined as the difference between epigenetic and chronological age. Positive values of EAA (that is epigenetic age is higher than the chronological age) indicate accelerated ageing and vice versa. Since EAA could be correlated with chronological age and white blood cell (WBC) percentage, we computed the so-called 'intrinsic' EAA (Chen, Marioni, Colicino et al., 2016), defined as the residuals from the linear regression of EAA with chronological age and WBC percentages (Chen et al., 2016). The latter were estimated using the Houseman et al. (2012) algorithm. Intrinsic EAA is not dependent on age and WBC by definition. Ten individuals were missing on epigenetic age and are excluded from the analysis resulting in a final case base of 490 individuals.

\section{Measures of socio-economic position (SEP)}

\subsection{Life course social class trajectory}

The sample was selected based on life course occupational mobility. We considered using education but chose not to because the meaning of education varies for different birth cohorts and Ireland introduced two major educational reforms in 1967 and 1972 which means older cohorts would have been over-represented among those classified as less educated. Occupation is favoured as an indicator of SEP in life course research because it is an individual-level measure that is correlated with other measures of SEP (e.g. income, education, and environmental exposures), and is transferrable as the occupational position of the head of household can be used to describe the likely socioeconomic circumstances of dependents (Galobardes et al., 2006). During the household interview, participants were asked to report their father's highest occupational position and their own current occupational position (or highest paying occupation if retired). Childhood and adulthood occupation were coded using the Irish Central Statistics Office social class schema (described elsewhere) (McCrory et al., 2015) and aggregated to create 3 groups as follows: (1) Professional/Managerial (2) Non-manual/skilled manual (3) Semi-skilled/unskilled/never worked. The cross-classification of childhood and adulthood social class was then used to characterise intergenerational social mobility. The exclusion of those missing on social class in childhood or adulthood reduced the sample available for analysis to 5346, of whom 3861 provided a blood sample (Supplementary Table 1). Due to the high cost of performing DNA methylation analysis, we could select only 500 cases from the sample of 3861 available for analysis. We decided to sample 4 groups comprising 125 cases each based on respondents' life course social class trajectory as follows in order to facilitate statistical comparisons across groups:

(1) Stable high - inter-generationally stable in the professional/ managerial class.

(2) Stable low - inter-generationally stable in the semi-skilled/ unskilled class

(3) Steep upward mobility - inter-generationally upwardly mobile from semi-skilled/unskilled childhood class to professional/managerial adulthood class

(4) Steep downward mobility - inter-generationally downwardly mobile from professional/managerial childhood class to semi-skilled/ unskilled adulthood class.

Prior to selection, the sample was pre-stratified by social class trajectory and sex, and 125 cases were randomly selected from within each category. Only 49 respondents experienced steep downward mobility so we supplemented the downwardly mobile group by including an additional 76 cases who were downwardly mobile by one social class position: 35 were downwardly mobile from professional/managerial childhood class to non-manual/skilled manual adulthood class, and a further 41 were downwardly mobile from non-manual skilled manual childhood class to semi-skilled/unskilled adulthood class. Of the total number of eligible cases within each social class trajectory, they are represented in the following proportions: stable high (125/ $457=0.27)$, stable low $(125 / 513=0.24)$ steep upward mobility $(125$ / $429=0.29)$, downwardly mobile $(125 / 295=0.42)$ with the latter group being over-represented relative to the others.

\subsection{Education}

Education is frequently employed as a measure of SEP because it tends to be completed early in life before the onset of many chronic conditions thereby reducing the risk of reverse causation. Highest level of educational attainment is represented as a three-level categorical variable: primary, secondary, tertiary, corresponding to approximately 10,12 and 15 years of formal education completed in the Irish education system.

\subsection{Income tertiles}

Respondents were asked to report all income resulting from full or part-time employment, private or public pensions, and income from other social welfare transfers. Respondents who could not provide an exact figure for income were asked to estimate their income using a banded range: (a) $<€ 10,000$ (b) $€ 10,000-<€ 20,000$ (c) $€ 20,000-<€ 40,000$; (d) €40,000- <€70,000 and (e) $\geq € 70,000$. We imputed income for those who provided a banded figure $(n=78)$ and for those who were missing on income $(n=42)$ using age, sex, education, marital status, employment status, household composition, and 
geographic region, which was implemented using the imputation by chained equations (uvis) command in Stata 15.0. Household income tertiles were generated after imputing for income.

\section{Health outcome measure}

\subsection{Timed up and go (TUG)}

We used performance on the TUG as our objective measure of health. It measures the time taken in seconds to rise from a chair (appro x.46 cms from the ground), walk $3 \mathrm{~m}$, turn around, return, and sit down. It is frequently used in epidemiological studies as an objective measure of physical functioning as it involves the co-ordinated action of a number of different systems including the nervous, cardio-pulmonary, and musculo-skeletal systems; and serves as a powerful indicator of health and vitality in older adults (Bergland et al., 2017). A previous publication from the TILDA team has shown that TUG captures the components of frailty that become more common with age and identifies frail members of the population well based on the Fried frailty phenotype (AUC $=0.87$ ) (Savva et al., 2013). Four participants did not complete the TUG task reducing the effective sample size to $n=486$ for the mediation analyses.

\subsection{Statistical analysis}

We used Stata, version 15.0 (Stata, College Station, TX) for all analyses. The correlation of chronological age with AL and the epigenetic clocks was examined using Pearson's correlation coefficient. The age and sex adjusted association of AL and the 3 measures of EAA with each measure of SEP was estimated independently using linear regression. We report results for the measure of AL which included the medications data. Because the measures of SEP were categorical, we tested whether they were jointly significant using an omnibus F-test after running the regression. As AL and the epigenetic clocks are expressed on different scales, we converted them to standard scores (zscores) in the analysis to enable direct comparison of SEP associations across the different measures. We also report the unstandardized estimates to facilitate comparison with other studies. Mediation analysis was undertaken using the Karlson, Holm and Breen (KHB) Method (Kohler et al., 2011). It provides a decomposition of the effects of both continuous and discrete variables and provides analytically derived statistical tests for determining the significance of mediating variables. The first model shows the age and sex adjusted association of SEP with TUG while the second model shows the change in the magnitude of the association when the mediating variable is entered. It then tests whether the addition of the mediator leads to a statistically significant reduction in the strength of the SEP-TUG association.

\section{Results}

Table 1 describes the characteristics of the sample. The mean age was 62.2 years $(\mathrm{SD}=8.3$ ), and $50.2 \%$ were female. Table 2 shows high risk cut-points for each component of the AL index separately for men and women. As expected, the three epigenetic clocks were strongly correlated with age: Horvath $(r=0.75)$, Hannum $(r=0.75)$ Levine $(r=0.84)$, while AL was much more modestly correlated with age $(r=0.32)$. There was no significant association between childhood SEP and AL, but AL was strongly associated with contemporaneous measures of adulthood SEP including, adulthood social class $(\mathrm{F}(2$, $485)=6.94, \mathrm{p}=0.0011)$, highest level of educational attainment $(\mathrm{F}(2$, $484)=4.90, p=0.0078)$, and household income tertiles $(\mathrm{F}(2$, $485)=6.05, p=0.0025)$. Participants in lower socio-economic strata were characterised by a higher AL burden compared with their more socio-economically advantaged peers (Table 3 ). Those in the unskilled/ semi-skilled adulthood social class position scored $0.33(\mathrm{CI}=0.15$, 0.50; $\mathrm{p}<0.001$ ) of a SD unit higher on AL compared with those in the
Table 1

Descriptive characteristics of the sample $(n=490)$.

\begin{tabular}{|c|c|c|c|}
\hline & All Sample & Median & Min/Max \\
\hline & Mean (SD) or $n(\%)$ & & \\
\hline Age & $62.2(8.3)$ & 61.0 & $50 / 87$ \\
\hline Female & $246(50.2 \%)$ & & \\
\hline $\begin{array}{l}\text { Horvath's epigenetic age acceleration } \\
\text { (EAA) }\end{array}$ & $0.0(7.3)$ & 0.0 & $-18.7 / 34.1$ \\
\hline $\begin{array}{l}\text { Hannum's epigenetic age acceleration } \\
\text { (EAA) }\end{array}$ & $0.0(7.3)$ & 0.0 & $-21.6 / 29.5$ \\
\hline $\begin{array}{l}\text { Levine's epigenetic age acceleration } \\
\text { (EAA) }\end{array}$ & $0.0(5.2)$ & 0.0 & $-15.2 / 16.7$ \\
\hline $\begin{array}{l}\text { Allostatic Load (excluding } \\
\text { medications) }\end{array}$ & $3.39(2.26)$ & 3.0 & $0.0 / 10.0$ \\
\hline $\begin{array}{l}\text { Allostatic Load (including } \\
\text { medications) }\end{array}$ & $4.06(2.58)$ & 4.0 & $0.0 / 12.0$ \\
\hline \multicolumn{4}{|l|}{ Childhood Social Class } \\
\hline - Professional/Managerial & $204(41.6 \%)$ & & \\
\hline - Non-manual/Skilled manual & $40(8.2 \%)$ & & \\
\hline - Semi-skilled/unskilled & $246(50.2 \%)$ & & \\
\hline \multicolumn{4}{|l|}{ Adulthood Social Class } \\
\hline - Professional/Managerial & $248(50.6 \%)$ & & \\
\hline - Non-manual/Skilled manual & $32(6.5 \%)$ & & \\
\hline - Semi-skilled/unskilled & $210(42.9 \%)$ & & \\
\hline \multicolumn{4}{|l|}{ Social Class Trajectory } \\
\hline - Stable high & $123(25.1 \%)$ & & \\
\hline - Upwardly mobile & $125(25.5 \%)$ & & \\
\hline - Downwardly mobile & $121(24.7 \%)$ & & \\
\hline - Stable low & $121(24.7 \%)$ & & \\
\hline \multicolumn{4}{|l|}{ Highest educational attainment } \\
\hline - Tertiary & $208(42.5 \%)$ & & \\
\hline - Secondary & $168(34.4 \%)$ & & \\
\hline - Primary & $113(23.1 \%)$ & & \\
\hline - missing & 1 & & \\
\hline \multicolumn{4}{|l|}{ Income Tertile } \\
\hline - Highest & $164(33.4 \%)$ & & \\
\hline - Intermediate & $163(33.3 \%)$ & & \\
\hline - Lowest & $163(33.3 \%)$ & & \\
\hline Tug Time (seconds) & $8.81(2.38)$ & 8.29 & $4.82 / 35.2$ \\
\hline
\end{tabular}

professional/managerial position. The primary educated scored 0.34 $(\mathrm{CI}=0.12,0.56 ; \mathrm{p}=0.002)$ of a SD unit higher on AL compared with the tertiary educated, while those in the lowest third of the household income distribution scored $0.37(\mathrm{CI}=0.16,0.58 ; \mathrm{p}<0.001)$ of a SD unit higher compared with those in the top third. In stark contrast, the EAA measures were not significantly associated with either childhood or adulthood SEP. These relationships are depicted graphically in Supplementary Figures $1 \mathrm{a}-1 \mathrm{e}$. Table 4 reports the unstandardized coefficients for comparability with other studies.

The cross-classification of childhood and adulthood social class was used to explore the impact of different life course socio-economic trajectories on these age-related biomarkers. Participants who were upwardly mobile were not significantly different to those in the stable high position with respect to AL burden, while those who were downwardly mobile and stable low were characterised by a higher AL burden, scoring $0.21(\mathrm{CI}=-0.03,0.44$; n.s. $)$ and $0.38(\mathrm{CI}=0.14,0.61$; $\mathrm{p}=0.002$ ) of a SD unit higher respectively compared with the stable high. Once again, there were no significant associations between life course social mobility patterns and the EAA measures.

As the EAA measures were not structured according to SEP, they were omitted from the mediation analysis. Table 5 shows the results of the decomposition analysis where the various measures of adulthood SEP are treated as exogenous variables, performance on the TUG test represents the outcome variable and AL is the mediator. Column 1 shows the age and sex adjusted association of the adulthood measures of SEP with TUG, column 2 shows the extent to which the coefficients are attenuated when we add AL to the model, and column 3 shows the percentage of the SEP-TUG association mediated by AL. We see partial mediation of the SEP-TUG relationship across each measure when we 
Table 2

Descriptive statistics and high-risk cut points for individual biomarkers of AL score for men and women.

\begin{tabular}{|c|c|c|c|c|c|c|c|c|}
\hline Biomarker & Mean & Median & Min & Max & P25 & P75 & N (\%) dysregulated & N (\%) dysregulated (with meds) \\
\hline \multicolumn{9}{|l|}{ MEN } \\
\hline CRP (ug/ml) & 2.39 & 1.42 & 0.00 & 28.8 & 0.53 & 2.94 & $63(24.9)$ & $63(24.9)$ \\
\hline Interleukin 1-ra (pg/ml) & 111.6 & 44.9 & 2.74 & 1727.0 & 24.0 & 100.4 & $63(24.9)$ & $63(24.9)$ \\
\hline Interleukin $6(\mathrm{pg} / \mathrm{ml})$ & 3.3 & 1.95 & 0.01 & 28.6 & 0.01 & 4.85 & $62(24.5)$ & $62(24.5)$ \\
\hline Interleukin $8(\mathrm{pg} / \mathrm{ml})$ & 13.5 & 10.6 & 2.40 & 106.7 & 7.40 & 17.3 & $62(24.5)$ & $62(24.5)$ \\
\hline $\mathrm{SBP}(\mathrm{mmHg})$ & 139.7 & 136.5 & 127.5 & 209.0 & 127.5 & 149.5 & $62(24.5)$ & $124(49.0)$ \\
\hline DBP (mmHg) & 84.1 & 83.5 & 58.5 & 125.0 & 77.5 & 90.0 & $60(23.7)$ & $60(23.7)$ \\
\hline RHR (bpm) & 67.8 & 65.5 & 43.5 & 115.5 & 58.5 & 75.5 & $62(24.5)$ & $108(42.7)$ \\
\hline HDL (mmol/L) & 1.37 & 1.34 & 0.64 & 2.65 & $1.12^{*}$ & 1.53 & $65(25.7)$ & $65(25.7)$ \\
\hline Total cholesterol (mmol/L) & 4.87 & 4.90 & 2.40 & 8.77 & 4.19 & 5.60 & $60(23.7)$ & $137(54.2)$ \\
\hline Waist-hip ratio & 0.96 & 0.96 & 0.77 & 1.16 & 0.92 & 1.00 & $63(24.9)$ & $63(24.9)$ \\
\hline BMI & 29.3 & 28.8 & 20.3 & 59.3 & 26.2 & 31.7 & $63(24.9)$ & $63(24.9)$ \\
\hline HbA1c (mmol/mol) & 33.5 & 32.0 & 19.0 & 65.0 & 30.0 & 35.0 & $56(22.1)$ & $59(23.3)$ \\
\hline Creatinine (umol/L) & 88.4 & 86.0 & 49.0 & 148.0 & 78.0 & 95.0 & $60(23.7)$ & $60(23.7)$ \\
\hline Cystatin C (mg/L) & 0.98 & 0.94 & 0.62 & 2.0 & 0.86 & 1.05 & $59(23.3)$ & $59(23.3)$ \\
\hline WOMEN & Mean & Median & Min & Max & P25 & P75 & N (\%) dysregulated & N (\%) dysregulated (with meds) \\
\hline CRP (ug/ml) & 2.66 & 1.45 & 0.00 & 57.7 & 0.49 & 2.95 & $61(24.7)$ & $61(24.7)$ \\
\hline Interleukin 1-ra (pg/ml) & 90.7 & 35.7 & 7.15 & 1215.4 & 23.1 & 63.4 & $61(24.7)$ & $61(24.7)$ \\
\hline Interleukin $6(\mathrm{pg} / \mathrm{ml})$ & 3.06 & 1.95 & 0.01 & 37.0 & 0.01 & 4.62 & $61(24.7)$ & $61(24.7)$ \\
\hline Interleukin $8(\mathrm{pg} / \mathrm{ml})$ & 12.6 & 11.1 & 3.59 & 46.0 & 8.19 & 15.3 & $61(24.7)$ & $61(24.7)$ \\
\hline SBP $(\mathrm{mmHg})$ & 129.5 & 128.0 & 88.5 & 196.0 & 114.5 & 140.5 & $61(24.7)$ & $109(44.1)$ \\
\hline DBP (mmHg) & 80.7 & 80.3 & 51.5 & 117.5 & 71.0 & 87.5 & $61(24.7)$ & $61(24.7)$ \\
\hline RHR (bpm) & 70.2 & 70.0 & 46.5 & 110.5 & 63.0 & 77.0 & $58(23.5)$ & $95(38.5)$ \\
\hline $\mathrm{HDL}(\mathrm{mmol} / \mathrm{L})$ & 1.71 & 1.66 & 0.79 & 3.6 & $1.38^{*}$ & 1.97 & $65(26.3)$ & $65(26.3)$ \\
\hline Total cholesterol' (mmol/L) & 5.41 & 5.30 & 2.8 & 8.1 & 4.7 & 6.0 & $60(24.3)$ & $123(49.8)$ \\
\hline Waist-hip ratio & 0.86 & 0.86 & 0.66 & 1.13 & 0.82 & 0.90 & $61(24.7)$ & $61(24.7)$ \\
\hline BMI & 28.3 & 27.7 & 17.2 & 50.7 & 24.7 & 31.8 & $61(24.7)$ & $61(24.7)$ \\
\hline $\mathrm{HbA1c}(\mathrm{mmol} / \mathrm{mol})$ & 33.0 & 32.0 & 20.0 & 89.0 & 30.0 & 34.0 & $60(24.3)$ & $61(24.7)$ \\
\hline Creatinine (umol/L) & 71.7 & 69.0 & 44.0 & 116.0 & 62.0 & 78.0 & 59 (23.9) & $58(23.5)$ \\
\hline Cystatin C (mg/L) & 0.96 & 0.92 & 0.64 & 1.81 & 0.82 & 1.06 & $58(23.5)$ & 59 (23.9) \\
\hline
\end{tabular}

* The sex specific cut-points for the determination of allostatic load is $>75$ th percentile of each biomarker with the exception of high density lipoprotein (HDL) for which the cut-point is $<=25$ th percentile.

add AL to the model. Among measures of SEP that were significantly associated with TUG, AL mediated between $3.1 \%-17.5 \%$ of the total effect, significantly so for those in the lowest vs highest income tertile, and at the $10 \%$ level for those with primary vs tertiary education.

\subsection{Sensitivity checks}

As a check on the robustness of the findings of adult SEP on AL to early life SEP, we re-ran the models controlling for childhood SEP, but

Table 3

Association of Socio-Economic Position (SEP) with standardized measures of Allostatic Load and Epigenetic Age Acceleration.

\begin{tabular}{|c|c|c|c|c|c|}
\hline & $\begin{array}{l}\text { Allostatic Load excluding meds (z- } \\
\text { score) }\end{array}$ & $\begin{array}{l}\text { Allostatic Load including meds (z- } \\
\text { score) }\end{array}$ & Horvath EAA (z-score) & Hannum EAA (z-score) & Levine EAA (z-score) \\
\hline & B $(95 \% \mathrm{CI})$ & B $(95 \% \mathrm{CI})$ & B $(95 \% \mathrm{CI})$ & B $(95 \% \mathrm{CI})$ & B $(95 \% \mathrm{CI})$ \\
\hline \multicolumn{6}{|l|}{ Childhood social class } \\
\hline Professional/Managerial & $R E F$ & $R E F$ & $R E F$ & $R E F$ & $R E F$ \\
\hline Non-manual/skilled manual & $0.14(-0.19,0.47)$ & $0.13(-0.19,0.45)$ & $-0.18(-0.52,0.16)$ & $-0.09(-0.43,0.26)$ & $-0.10(-0.44,0.24)$ \\
\hline Semi-skilled/unskilled & $0.11(-0.08,0.29)$ & $0.09(-0.09,0.26)$ & $0.11(-0.08,0.30)$ & $0.13(-0.06,0.31)$ & $0.07(-0.12,0.25)$ \\
\hline \multicolumn{6}{|l|}{ Adulthood social class } \\
\hline Professional/Managerial & $R E F$ & $R E F$ & $R E F$ & $R E F$ & $R E F$ \\
\hline Non-manual/skilled manual & $0.16(-0.19,0.52)$ & $0.18(-0.17,0.52)$ & $0.08(-0.29,0.45)$ & $0.11(-0.27,0.48)$ & $0.01(-0.36,0.38)$ \\
\hline Semi-skilled/unskilled & $0.33^{* * * *}(0.16,0.51)$ & $0.33^{\text {***k }}(0.15,0.50)$ & $0.01(-0.18,0.19)$ & $0.03(-0.15,0.22)$ & $0.05(-0.13,0.23)$ \\
\hline \multicolumn{6}{|l|}{ Social class trajectory } \\
\hline Stable High & $R E F$ & $R E F$ & $R E F$ & $R E F$ & $R E F$ \\
\hline Upwardly mobile & $-0.01(0.25,0.23)$ & $-0.03(-0.26,0.20)$ & $0.01(-0.24,0.26)$ & $0.08(-0.17,0.33)$ & $0.11(-0.14,0.36)$ \\
\hline Downwardly mobile & $0.22(-0.02,0.46)$ & $0.21(-0.03,0.44)$ & $-0.11(-0.36,0.14)$ & $-0.02(-0.28,0.23)$ & $0.07(-0.19,0.32)$ \\
\hline Stable low & $0.40^{* * * *}(0.16,0.64)$ & $0.38^{* *}(0.14,0.61)$ & $0.16(-0.09,0.41)$ & $0.19(-0.07,0.44)$ & $0.13(-0.12,0.38)$ \\
\hline \multicolumn{6}{|l|}{ Educational attainment } \\
\hline Tertiary & $R E F$ & $R E F$ & $R E F$ & $R E F$ & $R E F$ \\
\hline Secondary & $0.05(-0.14,0.25)$ & $0.06(-0.13,0.25)$ & $-0.08(-0.28,0.13)$ & $-0.16(-0.37,0.04)$ & $-0.04(-0.25,0.16)$ \\
\hline Primary & $0.33^{* *}(0.10,0.55)$ & $0.34 * *(0.12,0.56)$ & $0.00(-0.24,0.23)$ & $-0.06(-0.29,0.18)$ & $0.10(-0.13,0.33)$ \\
\hline \multicolumn{6}{|l|}{ Income Tertile } \\
\hline Highest & $R E F$ & $R E F$ & $R E F$ & $R E F$ & $R E F$ \\
\hline Intermediate & $0.19(-0.02,0.41)$ & $0.23^{*}(0.02,0.44)$ & $-0.07(-0.29,0.15)$ & $-0.15(-0.37,0.07)$ & $0.04(-0.18,0.27)$ \\
\hline Lowest & $0.31 * *(0.10,0.53)$ & $0.37^{* * * *}(0.16,0.58)$ & $0.08(-0.15,0.30)$ & $-0.01(-0.23,0.22)$ & $0.14(-0.08,0.37)$ \\
\hline
\end{tabular}

Age and sex adjusted independent association of measures of SEP with AL and the epigenetic clocks.

${ }^{* * *}$ significant at the 0.001 level; ** significant at the 0.01 level; * significant at the 0.05 level. 
Table 4

Association of Socio-Economic Position (SEP) with unstandardized measures of Allostatic Load and Epigenetic Age Acceleration.

\begin{tabular}{|c|c|c|c|c|c|}
\hline & Allostatic Load excluding meds & Allostatic Load including meds & Horvath EAA & Hannum EAA & LevineEAA \\
\hline & B $(95 \% \mathrm{CI})$ & В $(95 \% \mathrm{CI})$ & B $(95 \% \mathrm{CI})$ & B $(95 \% \mathrm{CI})$ & В $(95 \% \mathrm{CI})$ \\
\hline \multicolumn{6}{|l|}{ Childhood social class } \\
\hline Professional/Managerial & REF & $\mathrm{REF}$ & REF & REF & REF \\
\hline Non-manual/skilled manual & $0.32(-0.43,1.07)$ & $0.33(-0.50,1.17)$ & $-1.34(-3.84,1.17)$ & $-0.63(-3.10,1.85)$ & $-0.54(-2.32,1.24)$ \\
\hline Semi-skilled/unskilled & $0.24(-0.17,0.65)$ & $0.23(-0.23,0.68)$ & $0.80(-0.56,2.17)$ & $0.93(-0.42,2.28)$ & $0.35(-0.62,1.32)$ \\
\hline \multicolumn{6}{|l|}{ Adulthood social class } \\
\hline Professional/Managerial & REF & REF & REF & REF & REF \\
\hline Non-manual/skilled manual & $0.37(-0.43,1.17)$ & $0.46(-0.43,1.35)$ & $0.58(-2.14,3.30)$ & $0.77(-1.92,3.45)$ & $0.04(-1.89,1.97)$ \\
\hline Semi-skilled/unskilled & $0.76^{\text {****** }}(0.36,1.16)$ & $0.84^{* * * *}(0.40,1.29)$ & $0.06(-1.30,1.42)$ & $0.24(-1.10,1.58)$ & $0.26(-0.70,1.22)$ \\
\hline \multicolumn{6}{|l|}{ Social Class Trajectory } \\
\hline Stable High & $\mathrm{REF}$ & REF & REF & REF & REF \\
\hline Upwardly mobile & $-0.02(-0.56,0.52)$ & $-0.08(-0.68,0.52)$ & $0.11(-1.73,1.94)$ & $0.56(-1.26,2.37)$ & $0.55(-0.75,1.86)$ \\
\hline Downwardly mobile & $0.49(-0.05,1.04)$ & $0.53(-0.08,1.14)$ & $-0.80(-2.64,1.05)$ & $-0.17(-2.00,1.66)$ & $0.34(-0.97,1.66)$ \\
\hline Stable low & $0.90^{* * * * * *}(0.35,1.44)$ & $0.97 * *(0.37,1.58)$ & $1.16(-0.69,3.01)$ & $1.35(-0.48,3.17)$ & $0.68(-0.64,1.99)$ \\
\hline \multicolumn{6}{|l|}{ Educational attainment } \\
\hline Tertiary & REF & REF & REF & REF & REF \\
\hline Secondary & $0.12(-0.33,0.56)$ & $0.15(-0.35,0.64)$ & $-0.57(-2.07,0.93)$ & $-1.18(-2.66,0.30)$ & $-0.23(-1.29,0.84)$ \\
\hline Primary & $0.74 * *(0.23,1.25)$ & $0.88^{* *}(0.32,1.45)$ & $-0.03(-1.75,1.69)$ & $-0.40(-2.10,1.30)$ & $0.52(-0.70,1.74)$ \\
\hline \multicolumn{6}{|l|}{ Household Income Tertile } \\
\hline Highest & REF & REF & REF & REF & REF \\
\hline Intermediate & $0.44(-0.05,0.92)$ & $0.59 *(0.05,1.13)$ & $-0.50(-2.13,1.13)$ & $-1.08(-2.69,0.53)$ & $0.23(-0.93,1.38)$ \\
\hline Lowest & $0.71 * *(0.22,1.20)$ & $0.96^{* * * *}(0.41,1.50)$ & $0.56(-1.10,2.22)$ & $0.00(-1.65,1.63)$ & $0.75(-0.42,1.92)$ \\
\hline
\end{tabular}

Age and sex adjusted independent association of measures of SEP with AL and the epigenetic clocks.

significant at the 0.001 level; ** significant at the 0.01 level; * significant at the 0.05 level.

Table 5

Difference in TUG Speed (Secs) according to Socioeconomic Position (SEP) and Proportion of the Total Effect mediated by Allostatic Load $(n=486)$.

\begin{tabular}{|c|c|c|c|}
\hline & Model 1 & Model 2 & Mediation \\
\hline Adulthood social class & B $(95 \% \mathrm{CI})$ & B $(95 \% \mathrm{CI})$ & $\%$ \\
\hline Professional/Managerial & $R E F$ & $R E F$ & $R E F$ \\
\hline $\begin{array}{l}\text { Non-manual/skilled } \\
\text { manual }\end{array}$ & $0.13(-0.63,0.89)$ & $0.08(-0.68,0.84)$ & $39.4 \%$ \\
\hline Semi-skilled/unskilled & $0.96^{k * * * *}(0.58,1.34)$ & $0.87^{* * \times * k}(0.49,1.26)$ & $9.5 \%$ \\
\hline Allostatic Load & - & $0.11^{* *}(0.03,0.19)$ & - \\
\hline \multicolumn{4}{|l|}{ Social Class Trajectory } \\
\hline Stable High & $R E F$ & $R E F$ & $R E F$ \\
\hline Upwardly mobile & $-0.17(-0.69,0.34)$ & $-0.17(-0.68,0.35)$ & $2.1 \%$ \\
\hline Downwardly mobile & $0.57 *(0.05,1.10)$ & $0.51(-0.01,1.04)$ & $10.5 \%$ \\
\hline Stable low & $0.95^{* * * *}(0.44,1.47)$ & $0.85^{* *}(0.32,1.37)$ & $11.2 \%$ \\
\hline Allostatic Load & - & $0.11 * *(0.03,0.19)$ & - \\
\hline \multicolumn{4}{|l|}{ Educational attainment } \\
\hline Tertiary & $R E F$ & $R E F$ & $R E F$ \\
\hline Secondary & $0.57^{* *}(0.15,0.99)$ & $0.55^{*}(0.13,0.97)$ & $3.1 \%$ \\
\hline Primary & $0.89^{k * * * *}(0.40,1.38)$ & $0.78^{* *}(0.29,1.27)$ & $12.2 \%^{\mp}$ \\
\hline Allostatic Load & - & $0.13^{* * * * *}(0.05,0.20)$ & - \\
\hline \multicolumn{4}{|l|}{$\begin{array}{l}\text { Household Income } \\
\text { Tertile }\end{array}$} \\
\hline Highest & $R E F$ & $R E F$ & $R E F$ \\
\hline Intermediate & $0.13(-0.33,0.59)$ & $0.05(-0.41,0.52)$ & $58.1 \%$ \\
\hline Lowest & $0.68 * *(0.21,1.15)$ & $0.56 *(0.09,1.04)$ & $17.5 \% *$ \\
\hline Allostatic Load & & $0.13^{* *}(0.05,0.20)$ & - \\
\hline
\end{tabular}

Adjusted for age and sex.

**** significant at the 0.001 level; ** significant at the 0.01 level; * significant at the 0.05 level.

$¥$ significant at the 0.10 level.

the estimates were not materially affected (Supplementary Table 2), which shows that the adulthood associations are not simply due to the correlation of early life with later life SEP (e.g. Gustafsson et al., 2011). We also examined childhood health as a possible explanatory factor and found no differences in associations (Supplementary Table 2). Secondly, we calculated alternative life course socio-economic trajectories based on education (i.e. cross-classification of father's and respondent's educational attainment) and material circumstances (i.e. cross-classification of self-rated childhood circumstances (pretty well off, about average, poor) with income tertiles in later life). The results were entirely consistent with what we observed for social class. Participants in the stable low educational group (i.e. father and respondent's highest level of education $=$ primary $)$ scored $0.42(\mathrm{CI}=0.13,0.72 ; \mathrm{p}<.001)$ of a SD unit higher on AL compared with the stable high educational grouping, while the educationally downwardly and upwardly mobile ranked somewhere in-between. Similarly, those who reported growing up poor and who were in the lowest income tertile (i.e. stable low) in later life scored $0.52(\mathrm{CI}=0.12,0.92 ; \mathrm{p}<.01)$ of a SD unit higher compared with those who were pretty well off in early life and in the highest income tertile in later life (i.e. stable high). The downwardly mobile $(\beta=0.35, \mathrm{CI}=0.03,0.67 ; \mathrm{p}<.05)$ also had a significantly higher AL score compared with the stable high group based on this classification, while the upwardly mobile $(\beta=0.08, \mathrm{CI}=-0.23,0.40)$ were not significantly different to the stable high. There were no significant associations of these alternative social mobility patterns with EAA. Supplementary tables S3 and S5 report the sample composition based on life course educational and material circumstances respectively, while supplementary tables 4 and 6 report the results from these analyses respectively.

\section{Discussion}

We motivated this paper by noting that individuals from disadvantaged backgrounds experience higher levels of disease and morbidity and live shorter lives on average compared with their more advantaged peers. They represent an accelerated ageing cohort. This has sparked debate about the mechanisms through which the social environment gets biologically embedded in the tissues and organs of the body to precipitate the earlier ageing of those of low SEP. Given that we cannot directly manipulate social rank in human populations, cohort studies offer arguably the best vehicle for assessing the impact of SEP on health over a long span of years as they tend to be broad and omnibus in nature, collecting information at the social, behavioural, and increasingly the biological level, allowing us to examine the explanatory value of different theoretical frameworks.

Consistent with the results of previous studies (Seeman et al., 2010; Gruenewald et al., 2012; Castagné et al., 2018), we found that AL, a putative sub-clinical measure of physiological dysregulation across multiple organ systems was strongly socio-economically patterned by adulthood SEP. The social gradient in AL burden was evident by 
adulthood social class, education and income. Analysis of inter-generational social mobility patterns was insightful as it showed that those who were upwardly socially mobile had a similar AL burden to those who were stable high despite experiencing low SEP during childhood. In contrast, the downwardly mobile and the stable low had a higher AL burden compared with the stable high group, although only the latter contrast was statistically significant. Taken together, these results show that adulthood SEP predominates, at least with respect to AL. The result for the upwardly mobile group suggests that there are no physiological 'scarring effects' of low childhood SEP, while the higher AL burden for the downwardly mobile group illustrates that high SEP in childhood does not buffer one against worsening socio-economic circumstances in later life. The fact that the stable low group had the highest AL burden suggests that persistent low SEP is associated with a series of life course biological insults, the cumulative toll of which gradually erodes health.

The epigenetic clocks by contrast were not significantly associated with childhood or adulthood measures of SEP. The lack of association between SEP and EAA is not without precedent. Neither Austin et al. (2018) nor Hughes et al (2018) observed a significant association of various adulthood measures of SEP with the epigenetic clock estimators. However, both studies did report an association between childhood social class and EAA. It should be acknowledged that epigenetic ageing is not linear; the clock runs fastest in the first year of life and decreases gradually in a non-linear manner until about 20 years of age when the rate slows to a constant rate (Horvath and Raj, 2018), so this may represent a critical/sensitive period effect. In general, we also observed stronger associations between childhood social class and EAA compared with contemporaneous social class for those in the semiskilled/unskilled social class category. The correlation between genome-wide average DNA methylation (GWAM) in twin pairs is highest at birth ( 0.8 ), decreases with age during adolescence, and remains relatively stable at ${ }^{\sim} 0.4$ throughout adulthood (Li et al., 2018) so there is good reason to suspect that socio-economic influences on epigenetic machinery may be particularly strident at this stage in the life course. Nevertheless, it should be acknowledged that our understanding of how SEP affects these epigenetic processes is still rudimentary and additional studies are needed to determine whether the epigenome represents a mechanism for understanding biological embedding.

This study was designed to determine whether AL or the epigenetic clock represent plausible frameworks for understanding the biological embedding of social adversity. We found that AL was more strongly associated with the various measures of SEP than were the epigenetic clocks. In general, the social gradient was evident when AL was used as the dependent variable in the analysis, but either not present or less pronounced when the EAA measures were regressed on SEP. AL also partially mediated the association of SEP with an objective measure of health - performance on the TUG task. It is noteworthy that neither Horvath nor Hannum's clock were correlated with performance on the TUG task, although Levine's was (Supplementary Figure 2a). To address concerns that the results we obtained were not simply due to the use of TUG as the outcome measure, we also examined the association of AL and the epigenetic clocks with 2 other commonly used metrics of health in older populations.

Five objective measures: walking speed, exhaustion, physical inactivity, unintentional weight loss, and grip strength were used to construct a Fried frailty phenotype measure (Fried et al., 2001). Scores of $0,1-2$, and 3 or more, indicate that participants are non-frail, prefrail, or frail respectively. As only a small proportion of the sample were classified as frail, we combined the pre-frail (23.9) and frail (1.6\%) categories to create a binary variable indicating non/frail or pre-frail/ frail status. Disability was measured using the Activities of Daily Living (ADL's) scale. ADLs included difficulties with (1) dressing, (2) walking across a room, (3) bathing or showering, (4) eating, such as cutting up food, (5) getting in or out of bed, and (6) using the toilet. The total score on the measure ranges from 0 to 6 (mean $=0.11, \mathrm{SD}=0.46$, median $=0, \min =0, \max =4)$. We modelled the outcomes using binary logistic (frailty) and poisson regression (disability) as appropriate. Results were similar to those observed for TUG. AL burden was associated with significantly increased risk of frailty and disability; Levine's clock was associated with significantly increased risk of frailty only, while Horvath's and Hannum's clocks were not significantly associated with either measure. These results are shown in Supplementary Figures $2 \mathrm{~b}$ (frailty) and 2c (disability).

On the basis of the results from this study, it would appear then that AL represents the more promising framework for understanding the biological embedding of disease. This conclusion should not be interpreted as an unequivocal endorsement of AL however, as the AL framework has been subjected to considerable empirical and theoretical scrutiny (Dowd et al., 2009; Juster et al., 2010; Delpierre et al., 2016). One of the most frequent criticisms is that there is little consensus regarding the measurement and definition of AL which has been variously operationalised across studies (Juster et al., 2010), and researchers continue to debate the number and type of biological parameters that are integral to the concept. Others have argued that some of the markers represent clinical (e.g. BMI) rather than subclinical indicators of disease. Moreover, the AL index used in the present study does not include measures of neuroendocrine functioning, which are theorised to play a central role in the physiological cascade leading to biological dysregulation of inflammatory, cardiovascular and metabolic functioning.

It should also be acknowledged that the study's null findings on the lack of relation between childhood SEP and the epigenetic clocks represents something of an outlier in the literature. As a check on the robustness of our findings, we re-ran the analyses using father's education (supplementary Table 4) and self-reported childhood socio-economic circumstances, growing up before the age of 14 (pretty well off, about average, poor) (supplementary table 6 ) as independent variables in the analysis. These supplementary analyses essentially replicated the findings that we observed for childhood social class suggesting that the null findings are not simply due to measurement error in the reporting or coding of childhood social class. Nor is there anything immediately apparent about the ecology of childhood disadvantage in Ireland (e.g. universal healthcare, welfare) that can account for the null findings as the UK also had a similar (arguably better) system of supports that did not preclude Hughes et al. (2018) from reporting legacy effects of childhood SEP in their study. One possibility is that the epigenetic clocks are not particularly well calibrated for older participants (Zhang et al., 2017). The mean age of TILDA respondents was 62.2 years compared to a mean age of 58.4 in Hughes study and a maximum age of 55 in Austin's study. Future studies should be designed to examine the magnitude of prediction errors at different age ranges to address this possibility.

\subsection{Strengths and limitations}

It could be argued that the lack of association between SEP and the EAA measures is a consequence of insufficient statistical power as our sample comprised only 490 participants. However, two pieces of evidence militate against this explanation. Firstly, Austin et al. (2018) observed a significant association of childhood SEP with Horvath's clock in a smaller sample $(n=335)$ of Canadian participants, and secondly; the small sample size did not preclude us finding strong associations between SEP and AL. Another possibility is that our age range was more homogenous (50-97) compared with Austin's study and Hughes' (2018) study, which involved participants in the age range 15-55 and 28-98 years respectively. The epigenetic clock runs fastest in early life and slows in later life (Horvath and Raj, 2018) so this may have contributed to null findings with respect to the clocks in respect of adulthood SEP. Consistent with this interpretation, Hughes et al. (2018) noted that the youngest people in their sample were "substantially older in terms of DNA methylation than chronologically, whereas for the older participants the reverse was true" (p. 11). Differences in blood 
and/or tissue type may also account for the different patterns of results across studies. Austin et al. measured DNAm levels in monocytes using peripheral blood mononuclear cells (PBMC's), while Hughes et al. measured epigenetic ageing rates using whole blood and the Illumina $850 \mathrm{k}$ chip, the same as that used in the present study. We selected the sample on the basis of 4 equally sized groups, but the downwardly mobile were over-represented relative to their distribution in the population, which may introduce potential bias. Finally, the results of the mediation analysis should be interpreted with caution as the mediator (AL) and the outcome (TUG) were measured contemporaneously making so it is impossible to establish temporal precedence. Longitudinal studies will be the ultimate arbiter of such disputes.

These limitations are outweighed by the considerable strengths of our study. We examined the theoretical and empirical utility of AL and the epigenetic clocks for advancing our understanding of 'embodiment' using a sample of participants who were explicitly chosen for this purpose based on their intergenerational life course socio-economic trajectory. The design of the study also allowed us to examine critical period vs accumulation effects in the data. Another considerable strength is the deep phenotyping of the TILDA cohort with each participant completing a detailed clinical health assessment which was administered by training nursing staff using standardized operating protocols, so the clinical biomarkers and processing of bloods was done to a very high standard. Furthermore, the study included a 'second generation epigenetic clock' measure (Levine's DNAm Phenoage) which is a very recent innovation and has been shown to outperform the first generation of clocks in the prediction of many age-related diseases (Horvath and Raj, 2018).

\section{Conclusions}

AL and the epigenetic clock have both been touted to represent a potential mechanism for the biological embedding of social adversity. The results of this study, which directly compared the explanatory value of AL and 3 epigenetic clocks within a sample of participants specifically selected for this purpose suggests that the former may represent the stronger candidate for understanding the mechanisms through which social adversity gets "under the skin". The finding that those who were upwardly mobile were comparable in AL burden to those who experienced high SEP suggests that these processes are not immutable but are responsive to changing socio-economic circumstances over the life course.

\section{Author contributions}

CMC, GF, PV and RAK designed the study. Sample collection and processing were performed by PK, HA and SP. Statistical analyses were performed by CMC. All authors contributed to draft the manuscript and revised it critically. All authors approved the final form of the manuscript for submission.

\section{Declaration of interest}

The authors report no conflicts of interest.

\section{Acknowledgements}

Cathal McCrory is supported by the Health Research Board (HRB) of Ireland under an Emerging Investigator Award (EIA-2017-012). Giovanni Fiorito is supported by the Italian Institute for Genomic Medicine (IIGM). This work was also supported by the Lifepath grant to Paolo Vineis at Imperial College London (European Commission H2020 grant, Grant number: 633666). Funding for the TILDA project was supported by the Irish Government, the Atlantic Philanthropies, and Irish Life plc. The funders had no involvement in the study design, collection, analysis and interpretation of data, or authorship of the submitted work.

\section{Appendix A. Supplementary data}

Supplementary material related to this article can be found, in the online version, at doi:https://doi.org/10.1016/j.psyneuen.2019.02. 018.

\section{References}

Adler, N.E., Stewart, J., 2010. Health disparities across the lifespan: meaning, methods, and mechanisms. Ann. N. Y. Acad. Sci. 1186 (1), 5-23.

Aiello, A.E., Kaplan, G.A., 2009. Socioeconomic position and inflammatory and immune biomarkers of cardiovascular disease: applications to the panel study of income dynamics. Biodemography Soc. Biol. 55 (2), 178-205. https://doi.org/10.1080/ 19485560903382304.

Austin, M.K., Chen, E., Ross, K.M., McEwen, L.M., Maclsaac, J.L., Kobor, M.S., Miller, G.E., 2018. Early-life socioeconomic disadvantage, not current, predicts accelerated epigenetic aging of monocytes. Psychoneuroendocrinology 97, 131-134. https://doi. org/10.1016/j.psyneuen.2018.07.007.

Bergland, A., Jørgensen, L., Emaus, N., Strand, B.H., 2017. Mobility as a predictor of allcause mortality in older men and women: 11.8 year follow-up in the Troms $\emptyset$ study. BMC Health Serv. Res. 17 (1), 22. https://doi.org/10.1186/s12913-016-1950-0.

Castagné, R., Garès, V., Karimi, M., Chadeau-Hyam, M., Vineis, P., Delpierre, C., KellyIrving, M., 2018. Allostatic load and subsequent all-cause mortality: which biological markers drive the relationship? Findings from a UK birth cohort. Eur. J. Epidemiol. 33 (5), 441-458. https://doi.org/10.1007/s10654-018-0364-1.

Chen, B.H., Marioni, R.E., Colicino, E., Peters, M.J., Ward-Caviness, C.K., Tsai, P.C., et al., 2016. DNA methylation-based measures of biological age: meta-analysis predicting time to death. Aging 8 (9), 1844-1865. https://doi.org/10.18632/aging.101020.

Cunliffe, V.T., 2016. The epigenetic impacts of social stress: how does social adversity become biologically embedded? Epigenomics 8 (12), 1653-1669.

Delpierre, C., Barboza-Solis, C., Torrisani, J., Darnaudery, M., Bartley, M., Blane, D., et al., 2016. Origins of health inequalities: the case for Allostatic Load. Longit. Life Course Stud. 7 (1), 79-103.

Dowd, J.B., Simanek, A.M., Aiello, A.E., 2009. Socio-economic status, cortisol and allostatic load: a review of the literature. Int. J. Epidemiol dyp277.

Fiorito, G., Polidoro, S., Dugue, P.A., Kivimaki, M., Ponzi, E., Matullo, G., et al., 2017. Social adversity and epigenetic aging: a multi-cohort study on socioeconomic differences in peripheral blood DNA methylation. Sci. Rep. 7 (1), 16266. https://doi. org/10.1038/s41598-017-16391-5.

Francis, D.D., 2009. Conceptualizing child health disparities: a role for developmental neurogenomics. Pediatrics 124 (Supplement 3), S196-S202.

Fried, L.P., Tangen, C.M., Newman, A.B., Hirsch, Gottdiener, C., J, Seeman, T., et al., 2001. Frailty in older adults: evidence for a phenotype. J. Gerontol. A. Biol. Sci. Med. Sci. 56 (3), M146-M156. https://doi.org/10.1093/gerona/56.3.M146.

Galobardes, B., Shaw, M., Lawlor, D.A., Lynch, J.W., Davey Smith, G., 2006. Indicators of socioeconomic position (part 1). J. Epidemiol. Community Health 60 (1), 7-12. https://doi.org/10.1136/jech.2004.023531.

Gruenewald, T.L., Karlamangla, A.S., Hu, P., Stein-Merkin, S., Crandall, C., Koretz, B., Seeman, T.E., 2012. History of socioeconomic disadvantage and allostatic load in later life. Soc. Sci. Med. 74 (1), 75-83. https://doi.org/10.1016/j.socscimed.2011.09. 037.

Gustafsson, P.E., Janlert, U., Theorell, T., Westerlund, H., Hammarstrom, A., 2011. Socioeconomic status over the life course and allostatic load in adulthood: results from the Northern Swedish Cohort. J. Epidemiol. Community Health 65, 986-992.

Hannum, G., Guinney, J., Zhao, L., Zhang, L., Hughes, G., Sadda, S., et al., 2013. Genomewide methylation profiles reveal quantitative views of human aging rates. Mol. Cell 49 (2), 359-367. https://doi.org/10.1016/j.molcel.2012.10.016.

Hertzman, C., 2012. Putting the concept of biological embedding in historical perspective. Proc. Natl. Acad. Sci. 109 (Supplement 2), 17160-17167.

Horvath, S., 2013. DNA methylation age of human tissues and cell types. Genome Biol. 14 (10), R115. https://doi.org/10.1186/gb-2013-14-10-r115.

Horvath, S., Raj, K., 2018. DNA methylation-based biomarkers and the epigenetic clock theory of ageing. Nat. Rev. Genetics 19 (6), 371-384. https://doi.org/10.1038/ s41576-018-0004-3.

Houseman, E.A., Accomando, W.P., Koestler, D.C., Christensen, B.C., Marsit, C.J., Nelson, H.H., et al., 2012. DNA methylation arrays as surrogate measures of cell mixture distribution. BMC Bioinformatics 13, 86. https://doi.org/10.1186/1471-2105-13-86.

Hughes, A., Smart, M., Gorrie-Stone, T., Hannon, E., Mill, J., Bao, Y., et al., 2018. Socioeconomic Position and DNA Methylation Age Acceleration across the Lifecourse. Am. J. Epidemiol. https://doi.org/10.1093/aje/kwy155. kwy155kwy155.

Juster, R.-P., McEwen, B.S., Lupien, S.J., 2010. Allostatic load biomarkers of chronic stress and impact on health and cognition. Neurosci. Biobehav. Rev. 35 (1), 2-16.

Karlamangla, A.S., Singer, B.H., McEwen, B.S., Rowe, J.W., Seeman, T.E., 2002. Allostatic load as a predictor of functional decline. MacArthur studies of successful aging. J. Clin. Epidemiol. 55 (7), 696-710.

Kohler, U., Karlson, K.B., Holm, A., 2011. Comparing coefficients of nested nonlinear probability models. Stata J. 11 (3), 420-438.

Krieger, N., 2005. Embodiment: a conceptual glossary for epidemiology. J. Epidemiol. Community Health 59 (5), 350-355. https://doi.org/10.1136/jech.2004.024562.

Levine, M.E., Lu, A.T., Quach, A., Chen, B.H., Assimes, T.L., Bandinelli, S., et al., 2018. An epigenetic biomarker of aging for lifespan and healthspan. Aging 10 (4), 573-591. https://doi.org/10.18632/aging.101414. 
Li, S., Wong, E.M., Dugué, P.-A., McRae, A.F., Kim, E., Joo, J.-H.E., et al., 2018. Genomewide average DNA methylation is determined in utero. Int. J. Epidemiol. 47 (3), 908-916. https://doi.org/10.1093/ije/dyy028.

Lynch, J.W., Smith, G.D., Kaplan, G.A., House, J.S., 2000. Income inequality and mortality: importance to health of individual income, psychosocial environment, or material conditions. Br. Med. J. 320 (7243), 1200-1204.

Majnik, A.V., Lane, R.H., 2014. Epigenetics: Where Environment, Society and Genetics Meet.

Marmot, M., Wilkinson, R.G., 2001. Psychosocial and material pathways in the relation between income and health: a response to Lynch et al. Br. Med. J. 322 (7296), 1233.

Marmot, M., Allen, J., Bell, R., Bloomer, E., Goldblatt, P., 2012. WHO European review of social determinants of health and the health divide. Lancet 380 (9846), 1011-1029. https://doi.org/10.1016/s0140-6736(12)61228-8.

McCrory, C., Henretta, J.C., O'Connell, M.D., Kenny, R.A., 2015. Intergenerational occupational mobility and objective physical functioning in Midlife and older ages. J. Gerontol. B Psychol. Sci. Soc. Sci gbv084.

McEwen, B.S., 2008. Central effects of stress hormones in health and disease: understanding the protective and damaging effects of stress and stress mediators. Eur. J. Pharmacol. 583, 174-185.

Prag, P., Richards, L., 2018. Intergenerational social mobility and allostatic load in Great Britain. J. Epidemiol. Community Health. https://doi.org/10.1136/jech-2017210171. Published online ahead of print November 2018.

Robertson, T., Batty, G.D., Der, G., Fenton, C., Shiels, P.G., Benzeval, M., 2012. Is socioeconomic status associated with biological aging as measured by telomere length? Epidemiol. Rev. 35 (1), 98-111.

Robertson, T., Popham, F., Benzeval, M., 2014. Socioeconomic position across the lifecourse \& allostatic load: data from the West of Scotland Twenty-07 cohort study. BMC Public Health 14, 184

Savva, G.M., Donoghue, O.A., Horgan, F., O’Regan, C., Cronin, H., Kenny, R.A., 2013.
Using timed-up-and-go to identify frail members of the older population. J. Gerontol. (Series A). Biol. Sci. Med. Sci. 68 (4), 441-446. https://doi.org/10.1093/gerona/ gls190.

Seeman, T., Crimmins, E., Huang, M.-H., Singer, B., Bucur, A., Gruenewald, T., et al., 2004. Cumulative biological risk and socio-economic differences in mortality: MacArthur studies of successful aging. Soc. Sci. Med. 58 (10), 1985-1997.

Seeman, T., Epel, E., Gruenewald, T., Karlamangla, A., McEwen, B.S., 2010. Socio-economic differentials in peripheral biology: Cumulative allostatic load. Ann. N. Y. Acad. Sci. 1186 (1), 223-239.

Seeman, M., Merkin, S., Karlamangla, A., Koretz, B., Seeman, T., 2014. Social status and biological dysregulation: the "status syndrome" and allostatic load. Soc. Sci. Med. 118, 143-151. https://doi.org/10.1016/j.socscimed.2014.08.002.

Stringhini, S., Carmeli, C., Jokela, M., Avendaño, M., Muennig, P., Guida, F., et al., 2017. Socioeconomic status and the $25 \times 25$ risk factors as determinants of premature mortality: a multicohort study and meta-analysis of 1.7 million men and women. Lancet 389 (10075), 1229-1237.

Taylor, S.E., Repetti, R.L., Seeman, T., 1997. Health psychology: what is an unhealthy environment and how does it get under the skin? Annu. Rev. Psychol. 48, 411-447. https://doi.org/10.1146/annurev.psych.48.1.411.

Troyanskaya, O., Cantor, M., Sherlock, G., Brown, P., Hastie, T., Tibshirani, R., et al., 2001. Missing value estimation methods for DNA microarrays. Bioinformatics 17 (6), 520-525. https://doi.org/10.1093/bioinformatics/17.6.520.

Whelan, B.J., Savva, G.M., 2013. Design and methodology of the irish longitudinal study on ageing. J. Am. Geriatr. Soc. 61 (Suppl 2), S265-268. https://doi.org/10.1111/jgs. 12199.

Zhang, Y., Hapala, J., Brenner, H., Wagner, W., 2017. Individual CpG sites that are associated with age and life expectancy become hypomethylated upon aging. Clin. Epigenetics 9, 9. https://doi.org/10.1186/s13148-017-0315-9. 